\title{
DETERMINATION OF LEVELS OF SERUM ALBUMIN AND ABSOLUTE CD4+ CELL COUNTS IN HIV INFECTED INDIVIDUALS
}

\author{
Sigamani Sunitha1, Reddi Jagannadham²
}

${ }_{1}^{1}$ Assistant Professor, Department of General Medicine, Guntur Medical College and Government General Hospital, Guntur, Andhra

Pradesh, India.

${ }^{2}$ Assistant Professor, Department of General Medicine, Guntur Medical College and Government General Hospital, Guntur, Andhra Pradesh, India.

ABSTRACT
BACKGROUND
CD4+ cell counts and HIV RNA levels have been widely accepted as the most powerful prognostic indicators of HIV disease
progression. Recent studies have suggested that low levels of serum albumin are associated with rapid disease progression to
AIDS, AIDS associated mortality and all-cause mortality, independent of CD4 cell counts and HIV RNA titre.

\section{MATERIALS \& METHODS}

Study included 60 HIV/AIDS patients > 18 years of age, presenting to Government General Hospital, Guntur, during the period of September 2017 to November 2018. It was a Case Control Study. Controls were non HIV/AIDS individuals, age and sex matched with the patients. A detailed history, clinical examination and laboratory investigations were done. Repeat measurements on 3rd month follow up include Albumin, CD4+ cell count, Haemoglobin, Total and Differential WBC counts.

\section{RESULTS}

Males were more common and presentation at Decompensated Stage (C3) was seen most often. More people presented with a recent onset of illness ( $<3$ months). Most common opportunistic infection was pulmonary TB. Heterosexual behaviour was the most common risk factor seen in patients in the present study.

\section{CONCLUSION}

There was a strong direct correlation between CD4 count and Albumin in cases both at baseline and follow up indicating that albumin could be used as a surrogate marker for immunosuppression in HIV/AIDS patients. Regression analysis showed significant linear trend suggesting albumin as a variable is an important factor affecting change in CD4 count.

\section{KEY WORDS}

HIV/AIDS, CD4 Count, Serum Albumin, Correlation

HOW TO CITE THIS ARTICLE: Sunitha S, Jagannadham R. Determine the levels of serum albumin and absolute CD4+ cell counts in HIV infected individuals. J. Evolution Med. Dent. Sci. 2019;8(09):622-625, DOI: 10.14260/jemds/2019/137

\section{BACKGROUND}

Human immunodeficiency virus (HIV) is a lentivirus (a member of the retrovirus family) that causes acquired immunodeficiency syndrome (AIDS), a condition in humans in which progressive failure of the immune system allows life-threatening opportunistic infections and cancers to thrive. ${ }^{1}$

HIV infects vital cells in the human immune system such as helper T cells (specifically CD4+ T cells), macrophages, and dendritic cells. HIV infection leads to low levels of CD4+ T cells through three main mechanisms: First, direct viral killing of infected cells; second, increased rates of apoptosis in infected cells; and third, killing of infected CD4+ T cells by CD8 cytotoxic lymphocytes that recognize infected cells. When CD4+ T cell numbers decline below a critical level, cellmediated immunity is lost, and the body becomes progressively more susceptible to opportunistic infections. ${ }^{2}$

'Financial or Other Competing Interest': None.

Submission 05-01-2019, Peer Review 14-02-2019,

Acceptance 20-02-2019, Published 04-03-2019.

Corresponding Author:

Dr. Reddi Jagannadham,

Door No. 15-9-4, Flat No. G-1,

Seascape Apartment, Krishna Nagar,

Maharani Peta, Visakhapatnam-530002,

Andhra Pradesh, India.

E-mail: reddijagannadham67@gmail.com

DOI: $10.14260 /$ jemds/2019/137
CD4+ cell counts and HIV RNA levels have been widely accepted as the most powerful prognostic indicators of HIV disease progression. ${ }^{3}$

Use of these markers is wide spread in developed countries, but in developing countries they are not regularly obtained due to cost and technology constraints. While they remain as main clinical markers, they do not fully explain an individual's prognosis. ${ }^{4}$

There is a need to identify and establish efficacy of alternate prognostic markers of immunosuppression. Following few candidates have been proposed Total Lymphocyte Count, Serum Albumin, Albumin/Globulin Ratio, Haemoglobin, Haematocrit, CRP, DHEAS, IgA, $\beta 2$ microglobulin, p24 Antigen, CD8+ Cell Counts, Level Of CD38 On CD8+ Cells, Platelet Counts. 5

Low levels of serum albumin have been associated with higher rates of mortality in different acute and chronic conditions. ${ }^{6}$ Recent studies have suggested that low levels of serum albumin are associated with rapid disease progression to AIDS, AIDS associated mortality and all-cause mortality, independent of CD4 cell counts and HIV RNA titre. ${ }^{7}$

Serum Albumin Level could prove to be a very useful, cheap and easily available surrogate test for predicting severity of HIV infection and for pre-treatment assessment 8 $\&$ clinical monitoring of response to anti retro viral therapy 9 and as a predictor of survival. ${ }^{10}$ 
Objective of the present study was to determine the levels of serum albumin and absolute CD4+ cell counts in HIV infected individuals.

\section{MATERIALS \& METHODS}

Study included $60 \mathrm{HIV} /$ AIDS patients presenting to Government general hospital, Guntur during the period of September 2017 to November 2018.

\section{Study Design}

It was a Case Control Study.

Age and sex matched controls were used from normal population who were HIV negative and underwent similar investigations.

Method of collection of data: Patient's informed consent was taken.

A detailed history, clinical examination and laboratory investigations including Haemoglobin, Total And Differential WBC Counts, Haematocrit, Liver Function Tests with Serum Albumin Level, Renal Function Tests, CD4+ Cell Counts, Urinary Albumin \& HIV Elisa and Western Blot, HIV RNA estimation (Wherever possible) and Stool analysis (Wherever necessary) were done.

Repeat measurements on $3^{\text {rd }}$ month follow up include Albumin, CD4+ cell count, Haemoglobin, Total and Differential WBC counts.

\section{Inclusion Criteria}

HIV infected/AIDS patients > 18 years of age.

\section{Exclusion Criteria}

A. Any pre-existing hepatobiliary disease-causing decrease in albumin level.

B. Any pre-existing renal disease/ chronic kidney disease causing decrease in albumin level.

C. Any pre-existing gastrointestinal disease-causing decrease in albumin level.

D. Any clinical evidence of congestive cardiac failure.

E. Any clinical evidence of shock.

F. Any h/o burns in last 21 days.

A complete general and systemic physical examination was done in all cases. Peripheral blood was drawn from all the study subjects under aseptic biosafety precautions Albumin measured in serum (yellow-top tube) AND method used is bromocresol green Olympus AU2700 analyser.

CD4+ T lymphocyte counting was done in automated four-color BD - two laser FACS calibre with multiset software instrument using flow cytometry single platform technology.

\section{Ethical Clearance}

Institutional Ethical clearance was obtained prior to the start of the study.

\section{Statistical Analysis}

Data entry was done by Microsoft Excel 2010 version and analysis using SPSS 17 software. Numerical data was expressed as the mean \pm SD (Standard Deviation). Comparison between 2 means was performed by using the Student $\mathrm{t}$ test. Correlation was assessed using Pearson's correlation test. A p value less than 0.05 was considered statistically significant.

\section{RESULTS}

\section{Demographic Data}

Age distribution showed maximum number of cases between $31-40$ years of age $(39.8 \%)$ with mean age being $39.6 \pm 10.7$ years. Males constituted $70 \%$ of cases while females constituted $30 \%$ of cases. Study subjects were divided into social classes with most common being class 2 (Upper middle) (45\%). Mean body mass index (BMI) was 21.14 \pm 2.07 . Mean waist/hip ratio is $0.97 \pm 0.05$.

\section{Clinical Presentation}

- Most of the patients included in the study had been diagnosed recently in last three months: $58.3 \%(n=35)$.

- Most common presenting complaint of patients in this study was fever $30 \%$ followed by cough (18.3\%).

- Diarrhoea and Seizures were third most common presenting features at $10 \%$

- Also 3 patients (5\%) were found positive during admission and pre-operative work up. Significant weight loss was presenting complaint of 5 patients $(8.3 \%)$. Neck swelling, breathlessness and altered sensorium were present in decreasing order as presenting feature.

\section{Opportunistic Infections}

Present study showed opportunistic infections in $48.3 \%$ of total cases and most common was pulmonary tuberculosis (38.3\%) followed by candidiasis $(23.4 \%)$.

\section{Pattern of High-Risk Behaviour}

Most common high-risk behaviour was heterosexual contact (38.3\%) followed by history of blood transfusion in $30.1 \%$ $(19 / 60)$ and injectable drug use in 5 patients (8.3\%). None of the patients had history of men having sex with men.

\section{CDC Classification}

Total number of AIDS patients according to CDC categorization $=\mathrm{A} 3+\mathrm{B} 3+$ all in $\mathrm{C}=4+3+1+17+22=47 / 60$ (78.3\%).

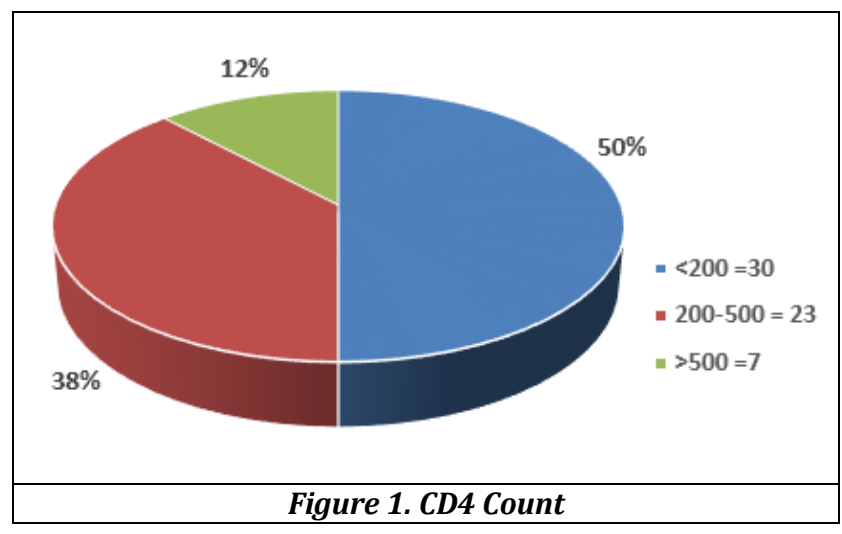

\section{Laboratory Profile}

The laboratory parameters were studied and showed no significant change in mean haemoglobin, albumin and CD 4 counts at baseline and third month follow up visit for both cases and controls. Analysis of differential counts of cases at baseline and follow up showed no significant variation. 


\begin{tabular}{|c|c|c|c|c|}
\hline Study Subjects & \multicolumn{2}{|c|}{ Cases } & \multicolumn{2}{c|}{ Controls } \\
\hline & Baseline & Follow Up & Baseline & Follow Up \\
\hline \multirow{2}{*}{ Haemoglobin } & $11.22 \pm$ & $11.23 \pm$ & $12.29 \pm$ & $12.34 \pm$ \\
& 2.08 & 2.01 & 1.58 & 1.48 \\
\hline \multirow{2}{*}{ Total Count } & $6885 \pm$ & $7038 \pm$ & $7143 \pm$ & $7207 \pm$ \\
& 2565 & 2190 & 1986 & 2116 \\
\hline \multirow{2}{*}{ CD4 Count } & $269.5 \pm$ & $268.67 \pm$ & $1004.7 \pm$ & $1008 \pm$ \\
& 187.5 & 179.7 & 232 & 228 \\
\hline Albumin & $3.02 \pm 0.6$ & $3.04 \pm 0.6$ & $4.26 \pm 0.8$ & $4.5 \pm 0.8$ \\
\hline \multicolumn{5}{|c|}{ Table 1. Laboratory Parameters } \\
\hline
\end{tabular}

Hence sub analysis was done after categorization of patients into low CD4 $(<200)$ and other category with count $>200$. It showed decrease in mean albumin on follow up in category with $\mathrm{CD} 4<200$.

Follow up showed a downward trend in the albumin levels in study patients from baseline levels in the category of patients with initial CD 4 counts $<200$. This was probably due to the low immune status of these patients and more time required for the stabilization of falling counts before a rising trend is noted.

For patients with CD4 counts $>200$ a linear an upward rising trend was present in the level of CD4 count at follow up.

\begin{tabular}{|c|c|c|}
\hline Albumin Levels & CD4 <200 & CD4 >200 \\
\hline Baseline & 2.85 & 3.19 \\
\hline Follow Up & 2.84 & 3.24 \\
\hline \multicolumn{2}{|c|}{ Table 2. Albumin and CD4 Levels in Cases } \\
\hline
\end{tabular}

\section{Correlation Between Albumin and CD4 Count in Cases and Controls}

Among cases, Albumin showed a direct correlation with CD4 count both at baseline $(\mathrm{p}<0.001)$ and during Follow up study $(\mathrm{p}<0.001)$ indicating that albumin could be used as a surrogate marker for immunosuppression in HIV/AIDS patients. (Correlation Coefficient $=0.52(\mathrm{p}<0.001)$.

Regression analysis showed significant linear trend suggesting albumin as a variable is an important factor affecting change in $\mathrm{CD} 4$ count $(\mathrm{p}=0.01)$

Such correlation was absent in the controls both at baseline and follow up.

\section{DISCUSSION}

Several studies have suggested that albumin could serve as a useful marker of HIV disease progression in resource-limited settings. In the present study, the relationship between serum albumin and absolute CD4+ cell count was studied in $60 \mathrm{HIV} /$ AIDS patients who presented to the Government General Hospital, Guntur during the period between September 2017 to November 2018.

Apart from descriptive analysis, correlation of CD4 count was done with serum albumin levels. Follow up was done in all 60 cases and correlation between albumin and CD4 count was done in these cases.

Mean age of patients in this study was $39.6 \pm 10.7$ years. Since the controls were age matched with patients, their means were similar. This is similar in comparison to other studies. Mehta et al $(2006)^{7}$ reported mean age of $39.9 \pm 11.3$ years and in Shah et al $(2007)^{4} 38.3 \pm 7.9$ years.

Male preponderance $(70 \%)$ was seen in present study which is in concurrence with Mehta et al7 (75.7\% males) and Kannangai et $\operatorname{al}^{5}$ (70.7\% males).
Kuppuswamy socioeconomic status is an important tool in hospital and community-based research in India. Socioeconomic parameters of subjects were studied and socioeconomic scoring in this study was done. Mean score was 15.17 with majority from upper middle (Class 2). None of the other studies had described a socioeconomic profile of study subjects.

The laboratory parameters were studied \& showed no significant change in mean haemoglobin, albumin and CD 4 counts at baseline and third month follow up visit for both cases and controls.

Follow up showed a downward trend in the albumin levels in study patients from baseline levels in the category of patients with initial CD4 counts $<200$. This was probably due to the low immune status of these patients and more time required for the stabilization of falling counts before a rising trend is noted.

For patients with CD4 counts $>200$ a linear an upward rising trend was present in the level of CD4 count at follow up.

The other studies had not used a control group for baseline determination of parameters in age and sex matched population.

In the study done by Mehta et $\mathrm{al}^{7}$ the effect of albumin before and after HIV seroconversion on disease progression was examined among individuals already infected with HIV at entry into a community-based cohort ( $n=453$ ) and further assessed among HIV seroconverters with albumin concentrations measured after $(n=219)$ and before seroconversion $(n=138)$. The prognostic effect of albumin on AIDS, AIDS mortality, and all-cause mortality was examined and among 453 HIV-infected individuals, albumin $<35 \mathrm{~g} /$ liter was associated with faster progression to AIDS [adjusted relative hazard (ARH), 1.8], AIDS mortality (ARH: 2.2), and all-cause mortality (ARH: 2.4). Analyses restricted to HIV seroconverters were similar. Pre-seroconversion levels of albumin did not predict outcomes, but HIV seroconversion appeared to lower albumin levels. These data showed that albumin $<35$ g/liter after HIV seroconversion was associated with faster HIV disease progression and suggested that low albumin levels are probably a consequence of HIV infection rather than merely reflective of some individuals inherently having low albumin levels. ${ }^{7}$

In the present study, the baseline and follow up values of albumin and CD4 counts were obtained and it was found to have a strong positive correlation amongst them, with correlation coefficient of 0.52 (baseline) and 0.51 (follow up) with both statistically significant $p$ values of $<0.001$ Such correlation was absent amongst the control group.

This suggests that serum albumin levels can be used as a surrogate marker for HIV disease monitoring as also showed in study by Oluwami. ${ }^{8}$

The differences found amongst HIV infected and normal control group were having strong statistical significance as also showed by study done in CMC Vellore by Kannangai. ${ }^{5}$

Though such strong correlation in short term (3 month) follow up used in this study is an indicator of usefulness of albumin as a surrogate marker but for further assessment of its effect on mortality needs a longer duration of follow up in a bigger study cohort as had been shown before in the study done by Mehta et al. 7 


\section{CONCLUSION}

Males were more commonly seen and presentation at Decompensated Stage (C3) was seen most often. More people presented with a recent onset of illness ( $<3$ months). Most common opportunistic infection was pulmonary TB. Heterosexual behaviour was the most common risk factor seen in patients of the present study. There was a strong and direct correlation between CD4 count and Albumin in cases both at baseline and follow up indicating that albumin could be used as a surrogate marker for immunosuppression in HIV/AIDS patients. Regression analysis showed significant linear trend suggesting albumin as a variable is an important factor along with change in CD4 count.

\section{REFERENCES}

[1] Kumar V, Abbas AK, Fausto N, et al. Robbins and Cotran Pathologic basis of disease. Chapter - 6 . Diseases of the immune system. Philadelphia, Pennsylvania: Elsevier 2010: p. 183-4.

[2] Cunningham AL, Donaghy H, Harman AN, et al. Manipulation of dendritic cell function by viruses. Current Opinion in Microbiology 2010;13(4):524-9.

[3] Kim S, Hughes MD, Hammer SM, et al. Both serum HIV type 1 RNA levels and CD4+ lymphocyte counts predict clinical outcome in HIV type 1-infected subjects with 200-500 CD4+cells per cubic millimetre. AIDS Res Hum Retroviruses 2000;16(7):645-53.
[4] Shah S, Smith CJ, Lampe F, et al. Haemoglobin and albumin as markers of HIV disease progression in the highly active antiretroviral therapy era: relationships with gender. HIV Med 2007;8(1):38-45.

[5] Kannangai R, Kandathil AJ, Ebenezer DL, et al. Usefulness of alternate prognostic serum and plasma markers for antiretroviral therapy for human immunodeficiency virus type 1 infection. Clin Vaccine Immunol 2008;15(1):154-8.

[6] Goldwasser P, Feldman J. Association of serum albumin and mortality risk. Journal of Clinical Epidemiology 1997;50(6):693-703.

[7] Mehta SH, Astemborski J, Sterling TR, et al. Serum albumin as a prognostic indicator for HIV disease progression. AIDS Research and Human Retroviruses 2006;22(1):14-21.

[8] Olawumi HO, Olatunji PO. The value of serum albumin in pre-treatment assessment and monitoring of therapy in HIV/AIDS patients. HIV Med 2006;7(6):351-5.

[9] Graham SM, Baeten JM, Richardson BA, et al. A decrease in albumin in early HIV type 1 infection predicts subsequent disease progression. AIDS Research and Human Retroviruses 2007;23(10):1197-200.

[10] Feldman JG, Burns DN, Gange SJ, et al. Serum albumin as a predictor of survival in HIV-infected women in the Women's Interagency HIV Study. AIDS 2000;14(7):863-70. 